\title{
Dependence of the stability of organic light-emitting diodes on driving mode
}

\author{
ZHANG WenWen ${ }^{1,2,3}$, WU ZhaoXin ${ }^{2,3 *}$, ZHANG XinWen ${ }^{2,3}$, LIANG ShiXiong $^{2,3}$, \\ JIAO $\mathrm{Bo}^{2,3} \&$ HOU Xun ${ }^{1,2,3}$ \\ ${ }^{1}$ State Key Laboratory of Transient Optics and Photonics, Xi'an Institute of Optics and Precision Mechanics, Chinese Academy of Sciences, Xi'an \\ 710119, China; \\ ${ }^{2}$ Key Laboratory for Physical Electronics and Devices of the Ministry of Education, School of Electronic and Information Engineering, Xi'an \\ Jiaotong University, Xi'an 710049, China; \\ ${ }^{3}$ Key Laboratory of Photonics Technology for Information of Shaanxi Province, School of Electronic and Information Engineering, Xi'an \\ Jiaotong University, Xi'an 710049, China
}

Received February 13, 2011; accepted April 1, 2011

\begin{abstract}
We investigated the effects of pulsed current (PC) and direct current (DC) driving modes on the stability of organic light-emitting diodes with and without hole-injection layers (HILs). Two different HIL materials were used: copper phthalocyanine (CuPc) and 4,4',4"-tris(3-methylphenylphenylamino)triphenylamine (m-MDTATA). It was found that the half-lives of devices using PC driving modes were different from those of comparable devices using DC driving modes. For the devices without HILs, with CuPc HILs and with MTDATA HILs, the half-lives of the devices were changed by factors of 1.91, 1.41 and 0.86 , respectively, when operated in PC rather than DC driving modes. Our analysis of the electrical characteristics of the corresponding hole-only devices showed that the number of holes injected into devices was greatly reduced by inserting an m-MTDATA layer compared with other designs. The results indicate that different ratios of injected electrons and holes can be obtained in these devices. Moreover, these ratios play a dominant role in the dependence of the stability of the device on the driving mode.
\end{abstract}

organic light-emitting diodes, stability, driving mode, electrons, holes

Citation: Zhang W W, Wu Z X, Zhang X W, et al. Dependence of the stability of organic light-emitting diodes on driving mode. Chinese Sci Bull, 2011, 56: 2210-2214, doi: 10.1007/s11434-011-4555-3

Organic light emitting diodes (OLEDs), which were first reported by Tang et al. in 1980s [1], have received a great deal of attention because of their applications in flat panel displays and lighting. The operational stability of OLEDs is a critical factor for commercial applications. Hence, many research groups have devoted resources to improving it. For small molecule-based OLEDs, several factors are believed to cause instabilities in these devices, such as electrochemical or thermal instabilities in the materials [2-4], charge imbalance between the injected holes and electrons [4-7], and instabilities at the material interfaces $[4,8]$. The driving method (direct current (DC) or pulsed current (PC)) is an

*Corresponding author (email: zhaoxinwu@mail.xjtu.edu.cn) important factor in determining the stability and lifetime of device. Previously [9-12], the lifetimes of OLEDs operating in PC mode were reported to be longer than those operating in DC mode. This was attributed to reasons such as the reduced speed of migration of ionic impurities in the OLEDs $[13,14]$, the reduction of the number of holes injected into the $\mathrm{Alq}_{3}$ layer by the reverse electric field of the $\mathrm{PC}$ driving mode [11]. As a result, the PC driving mode has been adopted in many OLED applications because of its long associated lifetime [9-12,15-17]. Recently, however, Luo et al. [18] found that the PC driving mode did not always lead to the longer lifetime for devices. For instance, the DC driving modes exhibited increased stability in comparison with PC driving mode with low duty cycles (4\%) and higher 
current densities $\left(27 \mathrm{~mA} / \mathrm{cm}^{2}\right)$. They also suggested that the dependence of device stability on the driving mode is correlated with its relative electroluminescence efficiency in PC and DC driving modes.

In this paper, we investigated the effect driving mode on the stability of OLEDs with different structures. We used a standard device based on N,N'-di(naphthalene-1-yl)-N, $\mathrm{N}^{\prime}$ diphenylbenzidine (NPB) and tris(8-hydroxyquinoline)aluminum $\left(\mathrm{Alq}_{3}\right)$. The other two OLEDs were trilayer devices based on $\mathrm{NPB} / \mathrm{Alq}_{3}$ with copper phthalocyanine $(\mathrm{CuPc})$ and 4,4',4"-tris(3-methylphenylphenylamino)triphenylamine (mMDTATA) serving as the hole-injection layers (HIL). It was found that the effect of the driving mode on the stability was not the same for all devices. The lifetimes of devices without a HIL and with CuPc as their HIL in PC operation were longer than that under DC operation. However, the opposite was true for devices with m-MDTATA serving as their HILs. We attribute different effects of these driving modes on the stability of the devices to different electronhole balances in their recombination zones; the balance is governed by the different ratios of injected electrons and holes in these devices.

\section{Experiments}

The devices were prepared via vacuum deposition onto indium tin oxide (ITO) coated glass substrates. The organic layers and cathode were deposited at a base pressure of about $1 \times 10^{-3} \mathrm{~Pa}$. The deposition rates were maintained at $0.2-0.3 \mathrm{~nm} / \mathrm{s}$ for the organic materials and $0.3-0.5 \mathrm{~nm} / \mathrm{s}$ for cathode material (aluminum). The structures are as follows:

Device A: ITO/NPB $(60 \mathrm{~nm}) / \mathrm{Alq}_{3}(60 \mathrm{~nm}) / \mathrm{LiF}(0.5 \mathrm{~nm}) /$ $\operatorname{Al}(100 \mathrm{~nm})$;

Device B: ITO/m-MTDATA (20 nm)/NPB (40 nm)/Alq 3 $(60 \mathrm{~nm}) / \mathrm{LiF}(0.5 \mathrm{~nm}) / \mathrm{Al}(100 \mathrm{~nm})$;

Device C: ITO/CuPc $(20 \mathrm{~nm}) / \mathrm{NPB}(40 \mathrm{~nm}) / \mathrm{Alq}_{3}(60 \mathrm{~nm}) /$ $\mathrm{LiF}(0.5 \mathrm{~nm}) / \mathrm{Al}(100 \mathrm{~nm})$.

In these devices, ITO, NPB, $\mathrm{Alq}_{3}$ and $\mathrm{LiF} / \mathrm{Al}$ are used as the anode, hole-transport layer (HTL), emitting/electrontransport layer (EML/ETL) and bilayer cathode, respectively. The m-MTDATA layer of device B and the CuPc layer of device $\mathrm{C}$ act as the HILs. The thickness of films was determined in situ with a quartz-crystal sensor and ex situ via profilometry (Nano-View MF-1000, Seoul, Korea). Electroluminescence and luminance characteristics were measured using a computer controlled programmable Keithley 2602 Source Meter (Cleveland, OH, USA) and a calibrated silicon photodiode. These devices were encapsulated in a dry nitrogen grove box. The measurements of stability were made using an adjustable constant current power source and a $50 \mathrm{~Hz}, 5 \mathrm{~V}$ reverse bias and $50 \%$ duty-cycle square-wave power source with an adjustable constant forward current. All measurements were performed at room temperature in normal laboratory conditions.

\section{Results and discussion}

Figure 1 shows the current density-voltage $(J-V)$, luminance-voltage $(L-V)$ and efficiency-voltage $(E-V)$ characteristics of devices A-C. Among all devices, device B had the highest luminance and current efficiency with the smallest current density. This indicates that the current efficiency and luminance can be effectively improved by inserting a HIL between the ITO anode and the HTL (NPB). Moreover, the m-MTDATA layer (device B) produced greater improvement than the CuPc layer (device $\mathrm{C}$ ).

Figure 2 shows the stability for devices $\mathrm{A}-\mathrm{C}$ when driven using the DC and PC modes. When the injected current in the reverse bias cycle of the PC mode is small, light emission is observed only during the forward bias cycle. The time-averaged injected current density is, therefore, half the value of the constant current density in the forward bias cycle [9]. The constant current density of the PC driving mode referenced in this article is the time-averaged current density. For device A, the lifetime testing was performed at constant current density of $44.56 \mathrm{~mA} / \mathrm{cm}^{2}$, which corresponds

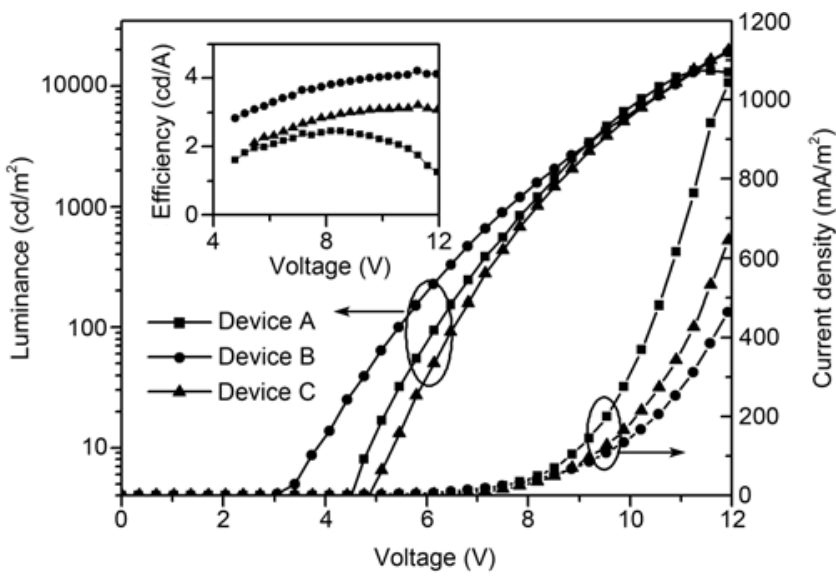

Figure 1 Current density-voltage and luminance-voltage characteristics for devices A, B and C. Inset: Current efficiency versus voltage characteristics for devices $\mathrm{A}, \mathrm{B}$ and $\mathrm{C}$.

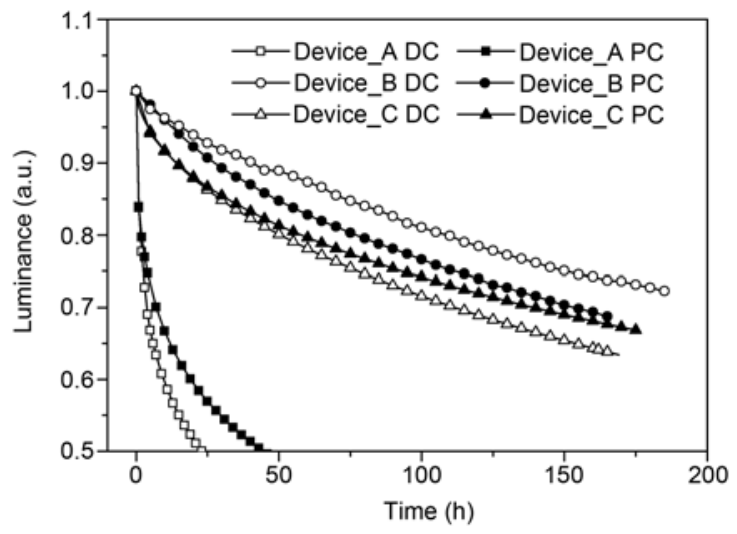

Figure 2 Luminance decay curves for devices A-C for both DC and PC. 
to initial luminance of $1013 \mathrm{~cd} / \mathrm{m}^{2}$ and $1025 \mathrm{~cd} / \mathrm{m}^{2}$ for the DC and PC modes. The half-life for PC operation was $44 \mathrm{~h}$, which is almost twice as long as that under DC driving $(23 \mathrm{~h})$. For device $\mathrm{C}$, the stability was measured at a constant current density of $31.65 \mathrm{~mA} / \mathrm{cm}^{2}$, which corresponds to the initial luminance of $1034 \mathrm{~cd} / \mathrm{m}^{2}$ and $1055 \mathrm{~cd} / \mathrm{m}^{2}$ for the DC and PC modes. The half-life for PC operation was $485 \mathrm{~h}$, which is about 1.5 times larger than that in the DC driving mode (345 h). However, for device B, the test current density was $31.19 \mathrm{~mA} / \mathrm{cm}^{2}$, which corresponds to the initial luminance of $1076 \mathrm{~cd} / \mathrm{m}^{2}$ and $1123 \mathrm{~cd} / \mathrm{m}^{2}$ for the DC and PC modes. In this case, PC operation led to lower stability than DC operation. The half-lives for device B in PC and DC operation were $391 \mathrm{~h}$ and $454 \mathrm{~h}$. The half-lives for devices $\mathrm{B}$ and $\mathrm{C}$ were extrapolated by fitting the measured decay curves with exponential decay function [19]:

$$
L(t)=L_{0} \exp \left[-\left(\frac{t}{\tau}\right)^{\beta}\right]
$$

where $L(t)$ is the luminance at time $t, L_{0}$ is the initial luminance, $\tau$ is corresponding to delay time, and $\beta$ is a dispersion factor. The results show that stability associated with each different driving mode depends on the device structure.

To investigate the essential differences among devices A-C, we fabricated a series of the hole-only devices. In these devices, the current density of the electrons was reduced to a negligible level via reducing the efficiency of the electron injecting contacts. The structure of these hole-only devices (device D) is: ITO/NPB $(120 \mathrm{~nm}) / \mathrm{Al}(100 \mathrm{~nm})$, device E: ITO/m-MTDATA $(20 \mathrm{~nm}) / \mathrm{NPB}(100 \mathrm{~nm}) / \mathrm{Al}$ $(100 \mathrm{~nm})$ and device F: ITO/CuPc $(20 \mathrm{~nm}) / \mathrm{NPB}(100 \mathrm{~nm}) /$ $\mathrm{Al}(100 \mathrm{~nm})$. Their energy levels are shown in Figure 3. Al was used as cathode material and has a Fermi energy of $4.28 \mathrm{eV}$. NPB was used as HTL and electron blocking layer and has a lowest unoccupied molecular orbital (LUMO) level of $2.6 \mathrm{eV}$. The large offset between the Fermi energy of the cathode and the LUMO level of NPB served to reduce the efficiency of electron injection and guarantee that holes injection dominates in the device [20]. Figure 4 shows the $J$ - $V$ characteristics of devices D-F (hole-only devices). Previously, it was reported that the CuPc HIL improved or reduced the hole injection efficiency depending on the experimental parameters and ITO treatment $[21,22]$. Device F had smaller hole current than device $\mathrm{D}$, which suggests the CuPc $(20 \mathrm{~nm})$ layer decreased hole injection efficiency in our devices. Device E had the smallest hole current, because the hole drift mobility of m-MTDATA less than those of NPB and CuPc [23]. Therefore, the number of holes injected into the device is reduced by inserting a HIL. In other words, the ratio of injected electrons and holes can be changed by inserting different HILs. In the standard bilayer $\mathrm{NPB} / \mathrm{Alq}_{3}$ device (device A), the hole mobility in NPB is almost two orders of magnitude higher than the electron mobility in $\mathrm{Alq}_{3}$ [24]. Thus, the HIL can be used to adjust

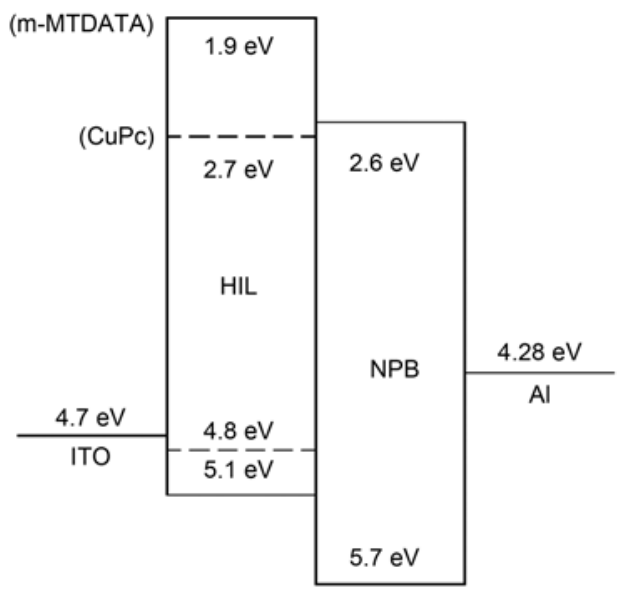

Figure 3 Energy level diagram for the hole-only devices D-F.

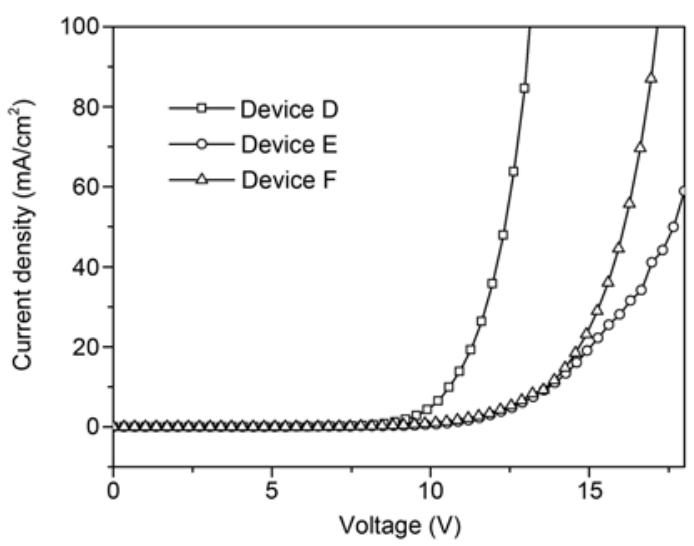

Figure 4 Current density-voltage characteristics for the hole-only devices D-F.

the ratio of injected electrons and holes in devices, reduce the accumulation of holes in the recombination zone, improve electron-hole balance in the recombination zone, and enhance device performance. This result is consistent with previous research [25,26]. Moreover, better electron-hole balance was expected when using an m-MTDATA HIL compared with that when using a CuPc HIL. Based on the above discussion, the essential difference among devices $\mathrm{A}-\mathrm{C}$ is their varying ratios of injected electrons and holes.

As mentioned above, for device $\mathrm{A}$, the number of holes is larger than that of electrons in the $\mathrm{NPB} / \mathrm{Alq}_{3}$ interface. Holes that are injected into the $\mathrm{Alq}_{3}$ layer may create unstable $\mathrm{Alq}_{3}$ cationic species during DC operation and the forward-biased period of PC operation. This is the main cause of device degradation [27]. However, some holes may drift back into the NPB layer because of the reverse-bias period of the PC driving mode. Therefore, PC operation exhibits a higher stability than DC operation. Device $\mathrm{C}$ has a better electron-hole balance than device A. Therefore, we expected that device $\mathrm{C}$ would have a minor improvement in the stability for PC operation in comparison with device A. Device B has the best electron-hole balance in the recombi- 
nation zone among the three devices. Even during DC operation, the leakage of holes into the $\mathrm{Alq}_{3}$ layer is substantially reduced because of the lower total hole current. Therefore, for device B the improvement in stability from the reverse bias period of PC operation is very limited. By contrast, the combinations of Joule heating and electricfield-induced disassociation of the singlet states, which are associated with the higher forward currents of PC operation, gradually become primary factors. This has a significant negative effect on the device stability. Because of the poor thermal conductivity of the glass substrate and organic materials, joule heating, which is caused by current injection and transport, occurs in the active area of the device. Consequently, the device temperature increases substantially. Higher device temperatures may result in thermal expansion, interlayer diffusion and crystallization in the organic layers, all of which accelerate the degradation of the devices. In addition, it was reported that NPB may also degrade because of electron injection [28]. The higher electric field caused by the higher forward current of PC driving mode can accelerate NPB degradation, and this may induce increased degradation in device $\mathrm{B}$ during $\mathrm{PC}$ operation. Consequently, the PC driving mode exhibits slightly lower stability compared to the DC driving mode for device B. Figure 5 shows the lifetime curves for device B for a highcurrent condition. The current density was $66.84 \mathrm{~mA} / \mathrm{cm}^{2}$, which corresponds to the initial luminance of $2422 \mathrm{~cd} / \mathrm{m}^{2}$ and $2436 \mathrm{~cd} / \mathrm{m}^{2}$ for the DC and PC operation. It was found that the stability for the PC driving mode is far smaller than that for the DC driving mode. This is in agreement with the theory that higher electric fields and Joule heating affect the degradation device B in the PC driving mode. From these results and discussion, we concluded that the varying ratios of injected electrons and holes in the devices result in different electron-hole balances in the recombination zone and effect of the driving mode on the stability shown in Figure 2.

\section{Conclusion}

In summary, the effect of the PC and DC driving modes on the operational stability of $\mathrm{NPB} / \mathrm{Alq}_{3}$-based OLEDs with three different structures (without HIL, CuPc HIL and mMTDATA HIL) was investigated. The effect of the driving mode on the stability depends on the type of device. Devices without HILs showed the expected improvement in device lifetime for PC driving modes in comparison with the DC driving mode. However, devices with a HIL showed minor improvement or a minor reduction in the device lifetime for the PC driving mode in comparison with the DC driving mode. These results were attributed to the varying ratios of injected electrons and holes in the devices. Adjusting the ratio of injected electrons and holes by inserting different HILs (or other methods), can improve the electronhole balance. In this case, the positive gains from PC driv-

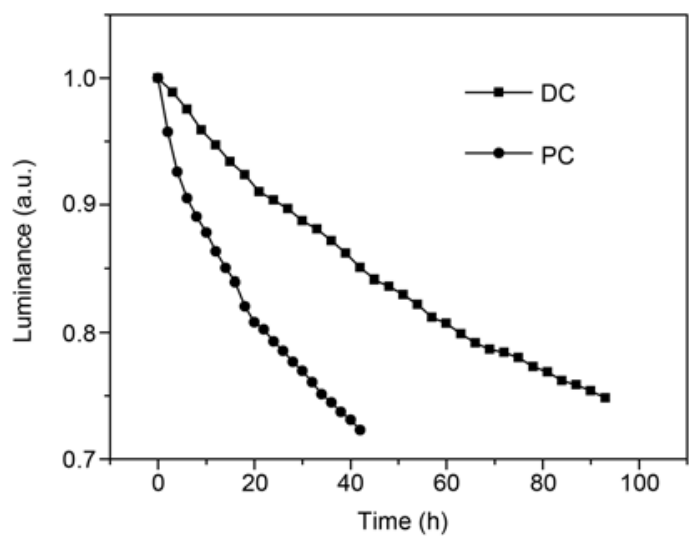

Figure 5 Luminance decay curves for device B for DC and PC operation in a high-current condition.

ing modes in the stability diminish, and negative effects associated with PC operation dominate. For a given OLED, we believe that the greatest operational stability can be achieved only through optimization of the driving mode.

This work was supported by the National Natural Science Foundation of China (10904122).

1 Tang C W, VanSlyke S A. Organic electro luminescent diodes. Appl Phys Lett, 1987, 51: 913-915

2 Fenter P, Schreiber F, Bulovi V, et al. Thermally induced failure mechanisms of organic light emitting device structures probed by X-ray specular reflectivity. Chem Phys Lett, 1997, 277: 521-526

3 Jiang H J. Effective adjustment of the optoelectronic properties of organic conjugated materials by synthesizing p-n diblock molecules. Chinese Sci Bull, 2011, 56: 119-136

4 Ishii M. Luminance decay mechanisms in organic light-emitting doides. R\&D Rev Toyota CRDL, 2003, 38: 55-60

5 Xu C, Sheng Y, Li Y, et al. Spectral variation of light-emitting diodes based on organic molecules doped polymer. Chinese Sci Bull, 2000, 45: 1817-1821

6 Li H J, Zhu R H, Yan L L, et al. Electroluminescence efficiency in bilayer organic light-emitting devices with LiF/Al cathode. Sci China Ser G-Phys Mech Astron, 2007, 50: 31-40

7 Vestweber H, Rie W. Highly efficient and stable organic light-emitting diodes. Synth Met, 1997, 91: 181-185

8 Lee S T, Gao Z Q, Hung L S. Metal diffusion from electrodes in organic light-emitting diodes. Appl Phys Lett, 1999, 75: 1404-1406

9 VanSlyke S A, Chen C H, Tang C W. Organic electroluminescent devices with improved stability. Appl Phys Lett, 1996, 69: 2160-2162

10 Pfeiffer M, Leo K, Zhou X, et al. Doped organic semiconductors: Physics and application in light emitting diodes. Org Electron, 2003, 4: 89-103

11 Cusumano P, Buttitta F, Di Cristofalo A, et al. Effect of driving method on the degradation of organic light emitting diodes. Synth Met, 2003, 139: 657-661

12 Li F, Feng J, Liu S. Degradation of organic light-emitting devices under different driving model. Synth Met, 2003, 137: 1103-1104

13 Zou D, Yahiro M, Tsutsui T. Improvement of current-voltage characteristics in organic light emitting diodes by application of reversed-bias voltage. Jpn J Appl phys, 1998, 37: L1406-L1408

14 Zou D, Yahiro M, Tsutsui T. Spontaneous and reverse-bias induced recovery behavior in organic electroluminescent diodes. Appl Phys Lett, 1998, 72: 2484-2486

15 Tsujioka T, Fujii H, Hamada Y, et al. Driving duty ratio dependence of lifetime of tris(8-hydroxy-quinolinate)aluminum-based organic 
light-emitting diodes. Jpn J Appl Phys, 2001, 40: 2523-2526

16 Tsujioka T, Hamada Y, Takahashi H. Operating current mode dependence of luminance properties of rubrene-doped yellow organic light emitting diodes. Jpn J Appl Phys, 2000, 39: 3463-3465

17 Aziz H, Popovic Z D. Degradation phenomena in small-molecule organic light-emitting devices. Chem Mater, 2004, 16: 4522-4532

18 Luo Y, Aziz H, Popovic Z D, et al. Correlation between electroluminescence efficiency and stability in organic light-emitting devices under pulsed driving conditions. J Appl Phys, 2006, 99: 054508

19 Zhang X, Wu Z, Wang D, et al. Improving the stability of organic light-emitting devices using a solution- processed hole-injecting layer. Appl Surf Sci, 2009, 255: 7970-7973

20 Wu Z, Wang L, Wang H, et al. Charge tunneling injection through a thin teflon film between the electrodes and organic semiconductor layer: Relation to morphology of the teflon film. Phys Rev B, 2006, 74: 165307

21 Forsythe E W, Abkowitz M A, Gao Y. Tuning the carrier injection efficiency for organic light-emitting diodes. J Phys Chem B, 2000, 104: 3948-3952

22 Divayana Y, Chen B J, Sun X W, et al. Hole injection or blocking? The role of CuPc in Alq3-based organic light-emitting devices. J
Cryst Growth, 2006, 288: 105-109

23 Tsang S W, Tong K L, Tse S C, et al. Direct determination of carrier mobilities of OLED materials by admittance spectroscopy. Proc SPIE, 2006, 6333: 633313

24 Martin S J, Verschoor G L B, Webster M A, et al. The internal electric field distribution in bilayer organic light emitting diodes. Org Elec, 2002, 3: 129-141

25 Wang H, Klubek K P, Tang C W. Current efficiency in organic lightemitting diodes with a hole-injection layer. Appl Phys Lett, 2008, 93 : 093306

26 Lu H T, Tsou C C, Yokoyama M. Organic light-emitting devices with energy-level aligned double-buffer layers. J Cryst Growth, 2006, 289: 161-163

27 Aziz H, Popovic Z D, Hu N X, et al. Degradation mechanism of small molecule-based organic light-emitting devices. Science, 1999, 283: 1900-1902

28 Kondakov D Y, Young R H. Variable sensitivity of organic lightemitting diodes to operation-induced chemical degradation: Nature of the antagonistic relationship between lifetime and efficiency. J Appl Phys, 2010, 108: 074513

Open Access This article is distributed under the terms of the Creative Commons Attribution License which permits any use, distribution, and reproduction in any medium, provided the original author(s) and source are credited. 